\title{
Direct versus indirect band gap emission and exciton-exciton annihilation in atomically thin molybdenum ditelluride $\left(\mathrm{MoTe}_{2}\right)$
}

\author{
Guillaume Froehlicher, Etienne Lorchat, and Stéphane Berciaud* \\ Institut de Physique et Chimie des Matériaux de Strasbourg and NIE, \\ UMR 7504, Université de Strasbourg and CNRS, \\ 23 rue du Loss, BP43, 67034 Strasbourg Cedex 2, France
}

\begin{abstract}
We probe the room temperature photoluminescence of $N$-layer molybdenum ditelluride $\left(\mathrm{MoTe}_{2}\right)$ in the continuous wave (cw) regime. The photoluminescence quantum yield of monolayer $\mathrm{MoTe}_{2}$ is three times larger than in bilayer $\mathrm{MoTe}_{2}$ and forty times greater than in the bulk limit. Monoand bilayer $\mathrm{MoTe}_{2}$ display almost symmetric emission lines at $1.10 \mathrm{eV}$ and $1.07 \mathrm{eV}$, respectively, which predominantly arise from direct radiative recombination of the A exciton. In contrast, $N \geq$ 3-layer $\mathrm{MoTe}_{2}$ exhibits a much reduced photoluminescence quantum yield and a broader, redshifted and seemingly bimodal photoluminescence spectrum. The low- and high-energy contributions are attributed to emission from the indirect and direct optical band gaps, respectively. Bulk $\mathrm{MoTe}_{2}$ displays a broad emission line with a dominant contribution at $0.94 \mathrm{eV}$ that is assigned to emission from the indirect optical band gap. As compared to related systems (such as $\mathrm{MoS}_{2}, \mathrm{MoSe}_{2}, \mathrm{WS}_{2}$ and $\mathrm{WSe}_{2}$ ), the smaller energy difference between the monolayer direct optical band gap and the bulk indirect optical band gap leads to a smoother increase of the photoluminescence quantum yield as $N$ decreases. In addition, we study the evolution of the photoluminescence intensity in monolayer $\mathrm{MoTe}_{2}$ as a function of the exciton formation rate $W_{\text {abs }}$ up to $3.6 \times 10^{22} \mathrm{~cm}^{-2} \mathrm{~s}^{-1}$. The lineshape of the photoluminescence spectrum remains largely independent of $W_{\mathrm{abs}}$, whereas the photoluminescence intensity grows sub-linearly above $W_{\text {abs }} \sim 10^{21} \mathrm{~cm}^{-2} \mathrm{~s}^{-1}$. This behavior is assigned to exciton-exciton annihilation and is well-captured by an elementary rate equation model.
\end{abstract}

PACS numbers: 78.67.-n, 71.35.Gg, 71.35.-y, 78.55.-m

\section{INTRODUCTION}

Transition metal dichalcogenides [1] (herein denoted $\mathrm{MX}_{2}$, where $\mathrm{M}=\mathrm{Mo}, \mathrm{W}, \mathrm{Re}$ and $\mathrm{X}=\mathrm{S}, \mathrm{Se}, \mathrm{Te}$ ) are an actively investigated class of layered materials, whose basic electronic, optical and vibrational properties depend critically on the number of layers $N$ that compose a given sample 26. $N$-dependent properties are remarkably illustrated by the transition from indirect optical band gap, in the bulk form, to direct optical band gap 7 at monolayer thickness that occurs in $2 \mathrm{Hc}$ Mo- and Wbased semiconducting $\mathrm{MX}_{2}$ [2, 3, 8, 11]. Direct optical band gaps, together with the possibility of achieving valley polarization for resonantly pumped band-edge excitons in monolayer $\mathrm{MX}_{2}$ 12, open original perspectives for two-dimensional optoelectronics [13] and valleytronics 14 .

An interesting direction in this field, consists in exploring $\mathrm{MX}_{2}$ with smaller optical band gaps (i.e., related to the formation of tightly bound excitons [1523) than the extensively studied monolayers of $\mathrm{MoS}_{2}$, $\mathrm{MoSe}_{2}, \mathrm{WS}_{2}, \mathrm{WSe}_{2}$, whose optical band gaps lie in the range $1.5-2.0 \mathrm{eV} 23$. Such endeavors are motivated by the possibility of achieving gate-controlled ambipolar transport more easily [24, 25] and to extend optoelectronic applications of $\mathrm{MX}_{2}$ and related van der Waals heterostructures 26] into the near-infrared range.

\footnotetext{
* stephane.berciaud@ipcms.unistra.fr
}

Among possible candidates, $N$-layer molybdenum ditelluride $\left(\mathrm{MoTe}_{2}\right)$ [6, 24, 25, 27, 33, as well as rhenium diselenide $\left(\mathrm{ReSe}_{2}\right)$ have emerged very recently. While $N$ layer $\mathrm{ReSe}_{2}$ crystals exhibit a distorted $1 T$-phase 3437 and are indirect optical band gap semiconductors, irrespective of $N$ 35, stable $N$-layer $2 \mathrm{Hc}-\mathrm{MoTe}_{2}$ crystals have been shown to undergo a transition from indirect (for bulk $\mathrm{MoTe}_{2}$ ) to direct (for monolayer $\mathrm{MoTe}_{2}$ ) optical band gap [27, 28. However, the exact value of $N$ at which the crossover occurs is a matter of debate 28 and a detailed analysis of the photoluminescence (PL) lineshape in $N$-layer $\mathrm{MoTe}_{2}$ is still lacking. In addition, the evolution of the PL spectrum and integrated PL intensity of monolayer $\mathrm{MoTe}_{2}$ with increasing exciton density remains unexplored so far.

In this article, we address the room temperature PL properties of $\mathrm{N}$-layer $2 \mathrm{Hc}$ - $\mathrm{MoTe}_{2}$ in the continuous wave (cw) regime. Our data show that the PL quantum yield of monolayer $\mathrm{MoTe}_{2}$ is approximately three times (forty times) larger than that of bilayer (bulk) $\mathrm{MoTe}_{2}$, confirming the transition from a bulk indirect optical band gap (giving rise to an emission line at $0.94 \mathrm{eV}$ ) to a direct optical band gap at $1.10 \mathrm{eV}$ [27. Moreover, an analysis of the PL lineshapes reveals two close-lying contributions to the PL spectra. For mono- and bilayer $\mathrm{MoTe}_{2}$, the observation of similar, almost symmetric PL spectra indicates that the crossover from dominant indirect to dominant direct band gap emission presumably occurs between $N=3$ and $N=2$ at room temperature. For $N=3$ to $N=7$ layers $\mathrm{MoTe}_{2}$, the low- and high-energy PL features are assigned to emission from the indirect 
and direct optical band gaps, respectively. Finally, the PL intensity of monolayer $\mathrm{MoTe}_{2}$ levels off with increasing laser intensity (i.e., as the exciton formation rate increases). This non-linear behavior unveils the critical role of exciton-exciton annihilation in atomically thin $\mathrm{MoTe}_{2}$, as also reported recently in other $\mathrm{MX}_{2}$ [22, 38, 42.

\section{METHODS}

$N$-layer crystals of trigonal prismatic (2Hc phase) $\mathrm{MoTe}_{2}$ (hereafter denoted $\mathrm{MoTe}_{2}$, see Fig. 1(a)) were prepared by mechanical exfoliation of commercially available bulk crystals (2D semiconductors) onto Si wafers covered with a $90 \mathrm{~nm}$-thick $\mathrm{SiO}_{2}$ epilayer (see Fig. 1(b)). The number of layers was first estimated from optical contrast and further confirmed by ultralow-frequency microRaman spectroscopy (see Fig. 11(c)-(d)). PL and Raman spectra were recorded in ambient conditions, both in a backscattering geometry, using a home-built setup. In Raman experiments, a combination of one narrow bandpass filter and two narrow notch filters (Optigrate) was used in order to attain the low-frequency range of the spectrum. After optimization, Raman features at frequencies as low as $4.5 \mathrm{~cm}^{-1}$ could be measured (see Fig. 1(c)). In all experiments, freshly prepared samples [31] were optically excited using a single longitudinal mode, linearly polarized, $2.33 \mathrm{eV}(532 \mathrm{~nm})$ laser beam focused onto $\mathrm{a} \approx 600 \mathrm{~nm}$-diameter spot using a high numerical aperture objective $(\mathrm{NA}=0.65)$. PL spectra in Figs. 2 3 were recorded in the linear regime at a laser intensity of approximately $1.5 \mathrm{~kW} / \mathrm{cm}^{2}$, using a single monochromator equipped with a 150 grooves $/ \mathrm{mm}$ ruled grating coupled to a thermoelectrically cooled twodimensional InGaAs array (Princeton Instruments NIRvana). Raman spectra were recorded at a laser intensity of approximately $60 \mathrm{~kW} / \mathrm{cm}^{2}$, using the same monochromator equipped with a 2400 grooves/mm holographic grating, coupled to a two-dimensional liquid nitrogen cooled charge-coupled device (CCD) array. We have verified that the higher laser intensities employed for Raman studies were not damaging our samples.

\section{DETERMINATION OF THE NUMBER OF LAYERS $N$}

Figure 1(c) shows the low-frequency Raman spectra (in the range $0-40 \mathrm{~cm}^{-1}$ ) of $N$-layer $\mathrm{MoTe}_{2}$, from $N=1$ to $N=7$, and of a thick sample $(N \gtrsim 50$ layers $)$ considered as a bulk reference. As previously reported [6], the low-energy features observed for $N \geq 2$ correspond to interlayer shear (LSM) and breathing (LBM) modes (see the gray dashed lines in Fig. 11(c)). In bulk $\mathrm{MoTe}_{2}$, the LBM is silent [43, 44] and only a single peak, assigned to the LSM can be observed. The evolution of the LSM and LBM frequencies with $N$ can be analytically described by the expression $\omega_{k}(N)=\frac{\omega_{0}}{\sqrt{2}} \sqrt{1-\cos \left(\frac{k \pi}{N}\right)}$
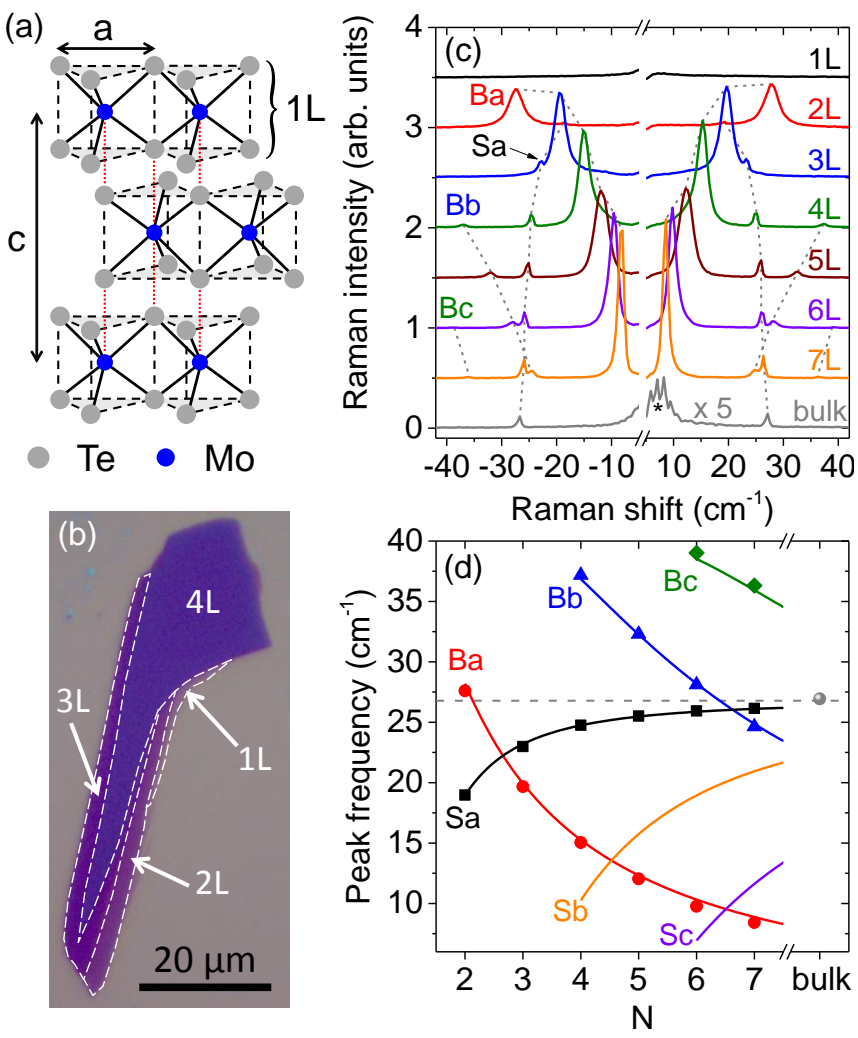

FIG. 1. (a) Side view of the crystal structure of $2 \mathrm{Hc}$ $\mathrm{MoTe}_{2}$. (b) Optical image of a $\mathrm{MoTe}_{2}$ flake (deposited onto a $\mathrm{Si} / \mathrm{SiO}_{2}$ substrate) containing mono to tetralayer domains. The boundaries of the various layers are highlighted with dashed lines. (c) Ultralow-frequency Raman spectra of $N=1$ to $N=7$ layer $\mathrm{MoTe}_{2}$ and of bulk $\mathrm{MoTe}_{2}$. The asterisk highlights residual contributions from the exciting laser beam. (d) Fan diagram of the interlayer shear ( $\mathrm{Sa}, \mathrm{Sb}$ and $\mathrm{Sc}$ ) and breathing $(\mathrm{Ba}, \mathrm{Bb}$ and $\mathrm{Bc})$ modes of $\mathrm{MoTe}_{2}$. Symbols are frequencies extracted from the Raman spectra in (c). The solid lines are theoretical calculations based on a linear chain model and the gray dashed line corresponds to the bulk frequency of the interlayer shear mode.

(with $k=1, \ldots, N-1$ ) deduced from a finite linear chain model 6, 44 47. Using this expression, the observed modes were fit using $k=N-1$ for the LSM branch (Sa) and $k=1,3,5$ for the LBM branches $(\mathrm{Ba}, \mathrm{Bb}$ and $\mathrm{Bc}$, respectively), as shown in Fig. 11(d). These fits yield bulk frequencies $\omega_{0}^{\mathrm{LSM}}=26.8 \mathrm{~cm}^{-1}$ and $\omega_{0}^{\mathrm{LBM}}=39.9 \mathrm{~cm}^{-1}$ in excellent agreement with the results in Ref. [6], further confirmed in Refs. 48, 49. This analysis permits an unambiguous determination of $N$.

\section{PL SPECTRA OF $N$-LAYER MoTe $\mathrm{M}_{2}$}

Figure 2(a) displays the raw PL spectra of the $\mathrm{MoTe}_{2}$ samples $(N=1$ to $N=7$ and bulk) previously introduced in Fig. 1. It is well known that interference effects strongly affect the exciton formation rate, as well 

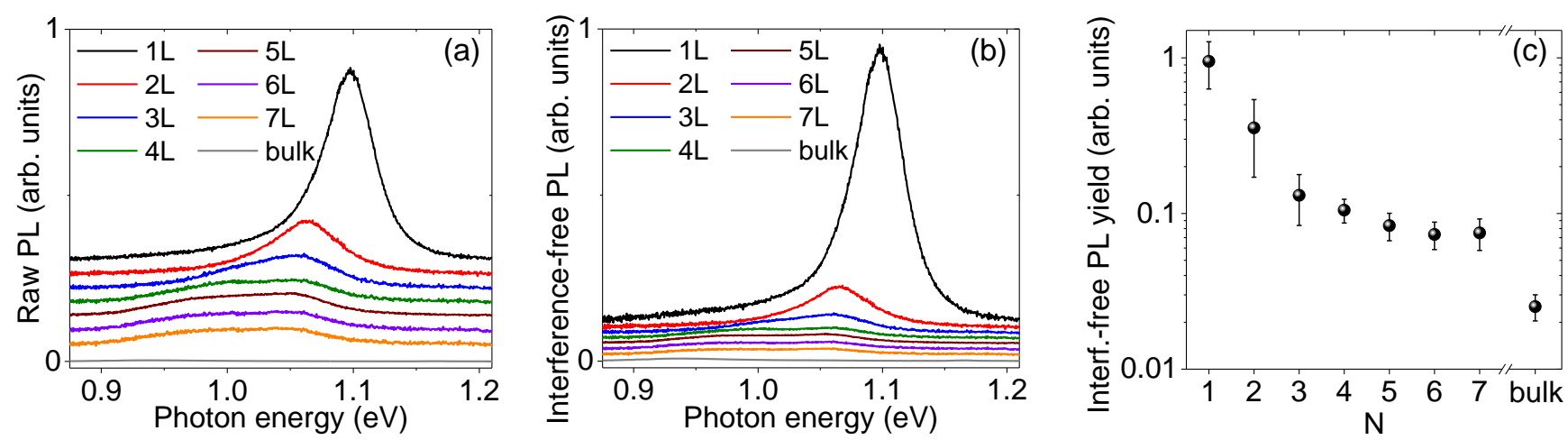

FIG. 2. (a) Raw and (b) interference-free photoluminescence spectra of $N=1$ to $N=7$ layer $\mathrm{MoTe}_{2}$ and of bulk MoTe 2 deposited on a $\mathrm{Si} / \mathrm{SiO}_{2}$ substrate. (c) Average total integrated intensities of the interference-free photoluminescence spectra as a function of $N$ obtained on three samples (except for $N=5$ and $N=6$, for which only one sample was studied).

as the Raman [50, 51] and PL 52] response of layered materials deposited on layered substrates such as $\mathrm{Si} / \mathrm{SiO}_{2}$. In order to take these phenomena into account, interference-free PL spectra were obtained by normalizing the raw spectra by the enhancement factor calculated following Refs. [50, 51] (see Fig. 2(b) and Supplemental Material 53). This procedure allows us to compare, in Fig. 2(c), the interference-free PL quantum yields, which are proportional to the integrated intensity of the interference-free PL spectra. Note that the enhancement factor takes into account the number of layers and is thus homogeneous to a length. Therefore, the interference-free PL quantum yields are given per unit length. Moreover contrary to what was reported in Ref. [29], the PL background from the $\mathrm{Si}$ substrate is negligible in our experiments (see Supplemental Material [53]).

Figure 2(b) displays the interference-free PL spectra. The PL lineshapes are marginally affected as compared to the raw spectra, whereas the integrated interference-free PL intensities are significantly modified. As $N$ increases, we immediately notice that (i) the integrated PL intensity decreases monotonically and is three (resp. forty) times smaller in bilayer (resp. bulk) $\mathrm{MoTe}_{2}$ than in the monolayer limit (see Fig. 2(c)), (ii) the PL peak energy redshifts from $1.10 \mathrm{eV}$ at monolayer thickness down to $0.94 \mathrm{eV}$ in the bulk limit and (iii) the PL lineshapes are slightly asymmetric for $N=1,2$ and clearly bimodal for $N \geq 3$. The first two observations are consistent with a transition from an indirect optical band gap in the bulk limit to a direct optical band gap for $N=1$ 27]. The increase in PL quantum yield as $N$ decreases is moderate, as compared to recent observations in $\mathrm{MoS}_{2}, \mathrm{MoSe}_{2}$, $\mathrm{WS}_{2}$, and $\mathrm{WSe}_{2}$ [2, 9, 10. This behavior is due to the smaller energy difference between the bulk emission from the indirect optical band gap and the direct optical band gap. For instance, the latter is approximately $0.6 \mathrm{eV}$ in $\mathrm{MoS}_{2}\left[2\right.$ and $0.5 \mathrm{eV}$ in $\mathrm{MoSe}_{2}[9]$.

\section{INDIRECT-TO-DIRECT OPTICAL BAND GAP CROSSOVER}

The exact value of $N$ at which the crossover occurs is still debated. At room temperature, Ruppert et al. 27. have suggested a crossover when reaching the monolayer limit, while at low temperature $(4-180 \mathrm{~K})$, Lezama et al. 28] concluded that the crossover occurs between $N=3$ and $N=2$. Very recently, at $10 \mathrm{~K}$, Robert et al. 54 have observed similar PL intensities in monoand bilayer $\mathrm{MoTe}_{2}$ and a slightly longer PL decay time in bilayer $\mathrm{MoTe}_{2}$ than in monolayer $\mathrm{MoTe}_{2}$, suggesting that PL in bilayer $\mathrm{MoTe}_{2}$ may in part originate from the direct optical band gap. However, there is no apparent contradiction between these claims since it is well-known that temperature might affect the crossover 8. Here, we could clearly identify two subfeatures within each PL spectrum, as illustrated in Fig. 3. We may now wonder whether these two contributions may be associated with the direct and indirect optical band gaps. To answer this question, we have systematically fit the PL spectra with a double Voigt profile (see Fig. 3) and extracted the high$\left(\mathrm{PL}^{+}\right)$and low-energy $\left(\mathrm{PL}^{-}\right)$contributions. Figure 4 displays the peak positions $\mathrm{PL}_{\max }^{+}$and $\mathrm{PL}_{\max }^{-}$.

First, the PL spectrum of monolayer $\mathrm{MoTe}_{2}$ exhibits an almost symmetric lineshape dominated by a relatively narrow $\mathrm{PL}^{+}$feature with a full width at half maximum (FWHM) of approximately $50 \mathrm{meV}$. The peak position $\mathrm{PL}_{\max }^{+}$matches the energy of the $\mathrm{A}$ exciton measured by room temperature differential reflectance spectroscopy by Ruppert et al. 27] (see Fig. 4(a)) and $\mathrm{PL}_{\max }^{+}$ is therefore identified as the direct optical band gap energy. The $\mathrm{PL}^{-}$shoulder is much broader (FWHM of approximately $100 \mathrm{meV}$ ) and has lower integrated intensity than that of the $\mathrm{PL}^{+}$peak. Assuming that monolayer $\mathrm{MoTe}_{2}$ is a direct optical band gap semiconductor, the $\mathrm{PL}^{-}$feature cannot arise from the indirect optical band gap. Since the energy difference between the $\mathrm{PL}^{ \pm}$ features is approximately $30 \mathrm{meV}$ (see Fig. 4(b)), the 


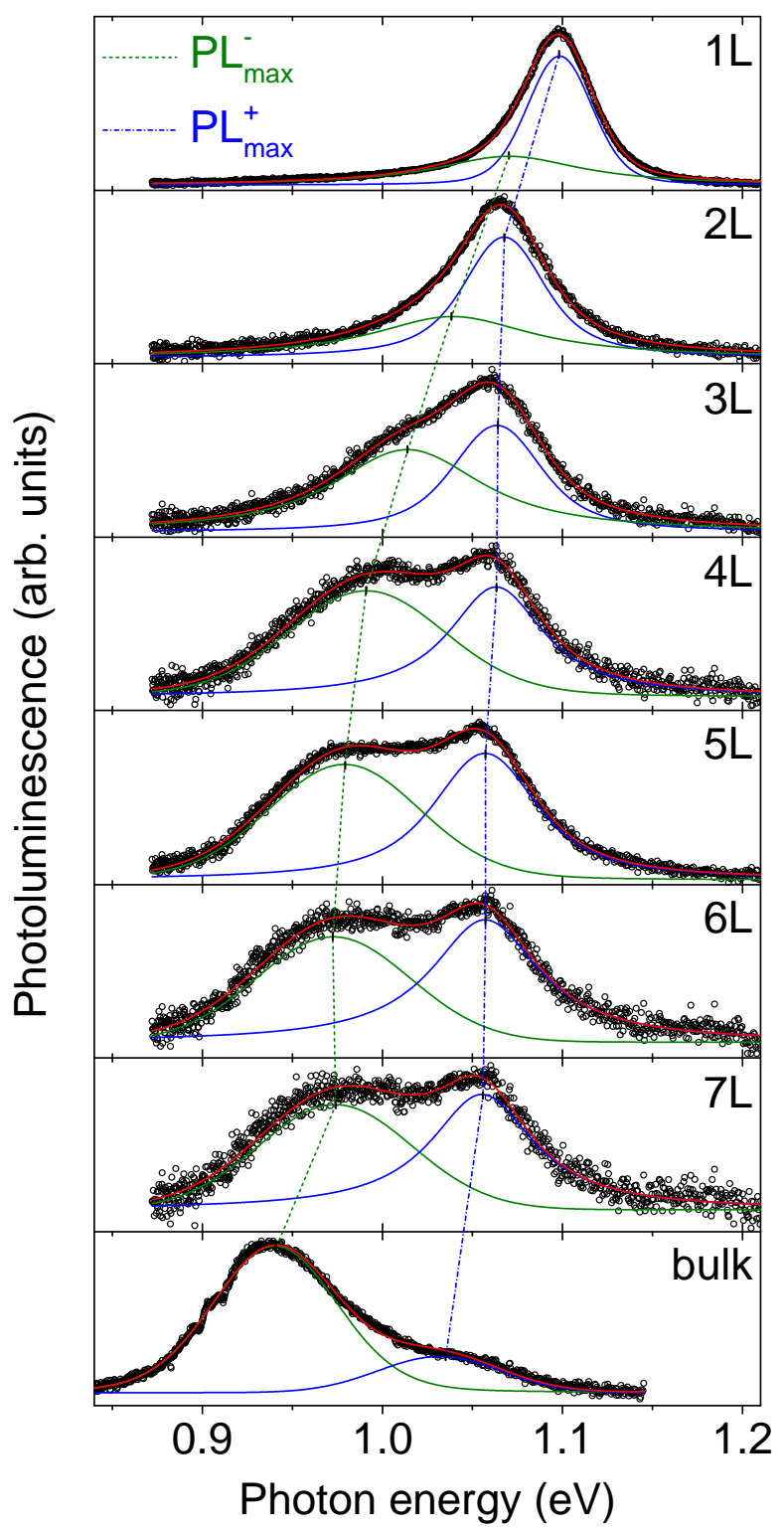

FIG. 3. Normalized interference-free photoluminescence spectra of $N=1$ to $N=7$ layer $\mathrm{MoTe}_{2}$ and of bulk MoTe 2 . The spectra are the same as in Fig. 2(b). The data (black open circles) are fit using the sum of two Voigt profiles (red solid lines). The green and blue solid lines are the $\mathrm{PL}^{-}$and $\mathrm{PL}^{+}$ features, respectively. The green dotted and blue dash-dotted lines mark the evolution of the associated peak energies, denoted $\mathrm{PL}_{\max }^{-}$and $\mathrm{PL}_{\max }^{+}$, respectively, as a function of the number of layers.

$\mathrm{PL}^{-}$peak can tentatively be assigned to emission from charged A excitons (i.e., trions [28, 29]) or to excitonphonon sidebands involving coupling of A excitons with $\Gamma$-point optical phonons (whose energies lie in the range 15-35 meV [6, 30]).

Second, the PL spectrum of bilayer $\mathrm{MoTe}_{2}$ is slightly redshifted (by about $30 \mathrm{meV}$ ) with respect to the mono-

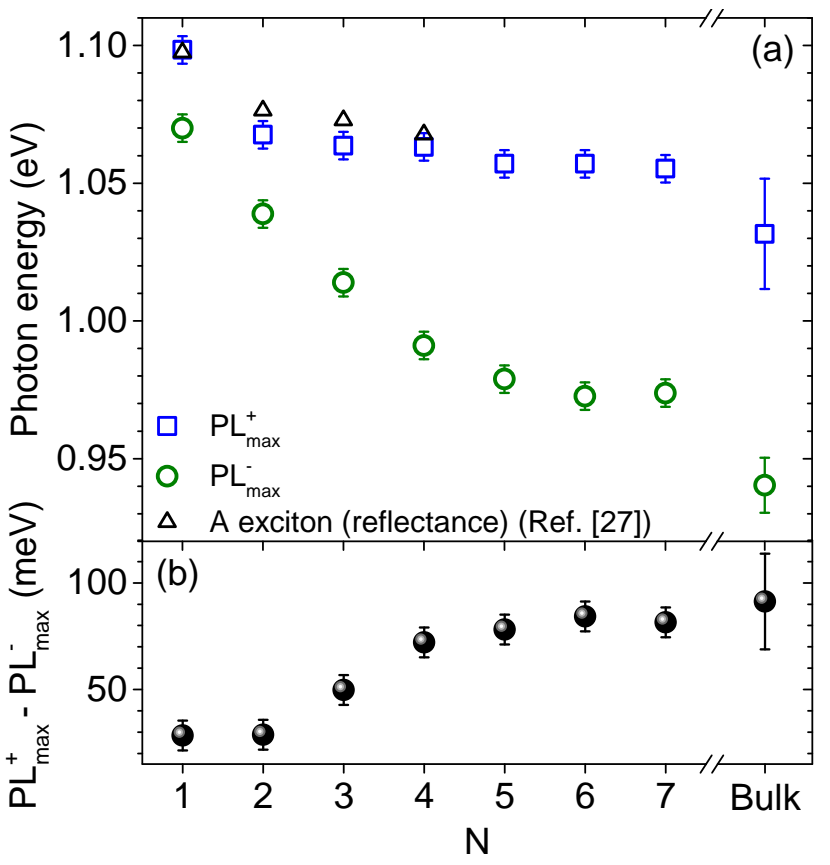

FIG. 4. (a) Energies of the photoluminescence peaks $\mathrm{PL}_{\max }^{-}$ (green open circles) and $\mathrm{PL}_{\max }^{+}$(blue open circles) as a function of the number of layers $N$. The data are extracted from the fits shown in Fig. 3 and correspond to the same samples as in Fig. 2(c). Our experimental measurements are compared to the reflectance measurements from Ref. [27] (open black triangles). (b) Energy difference between the two photoluminescence peaks as a function of the number of layers $N$.

layer case, with a normalized PL quantum yield about three times smaller than that of monolayer $\mathrm{MoTe}_{2}$, suggesting that bilayer $\mathrm{MoTe}_{2}$ is not a direct optical band gap semiconductor. However, although the bilayer PL spectrum is appreciably broader than that of the monolayer PL spectrum (FWHM of approximately $65 \mathrm{meV}$ ), the spectra are similar. Indeed, $\mathrm{PL}_{\max }^{+}$also matches the energy of the A exciton for $N=2$ [27]. In addition, the $\mathrm{PL}^{+}$peak is more intense than the $\mathrm{PL}^{-}$peak, and the energy difference between the peak positions of these two features remains approximately $30 \mathrm{meV}$ (see Fig. (4), as in monolayer $\mathrm{MoTe}_{2}$. These observations indicate that the room temperature PL in mono- and bilayer $\mathrm{MoTe}_{2}$ likely originates from similar mechanisms. However, the reduced PL quantum yield of bilayer $\mathrm{MoTe}_{2}$ suggests that the indirect optical band gap is slightly smaller than the direct optical band gap such that phonon-assisted emission across the indirect optical band gap may contribute to the broadening of the PL spectrum in bilayer $\mathrm{MoTe}_{2}$. Overall, we conclude that emission from the direct optical band gap dominates the room temperature PL response of bilayer $\mathrm{MoTe}_{2}$.

Third, the PL spectra of $N \geq 3$-layer $\mathrm{MoTe}_{2}$ differ markedly from the mono- and bilayer cases. We observe (i) a broad and prominent $\mathrm{PL}^{-}$feature (with a FHWM 
of approximately $100 \mathrm{meV}$ ), which, as $N$ increases, progressively dominates the narrower $\mathrm{PL}^{+}$feature (with a FWHM in the range 60-70 meV), and (ii), as $N$ increases, $\mathrm{PL}_{\max }^{-}$downshifts significantly, while $\mathrm{PL}_{\max }^{+}$remains almost constant and very close to the energy of the A exciton absorption line [27. In the bulk limit, the $\mathrm{PL}^{-}$ peak is centered at $0.94 \mathrm{eV}$ and is followed by a much fainter feature near $1.03 \mathrm{eV}$ [55]. Thus, the $\mathrm{PL}^{+}$and $\mathrm{PL}^{-}$ peaks can tentatively be assigned to competing emission pathways, associated with hot luminescence from the A exciton and with phonon-assisted emission from the indirect excitons, respectively. Note that the $\mathrm{PL}^{-}$peak is broader than the $\mathrm{PL}^{+}$peak, presumably due to the phonons involved in the indirect emission process. Finally, our conclusions are further confirmed by the fact that the bulk values of $\mathrm{PL}_{\max }^{+}$and $\mathrm{PL}_{\max }^{-}$are in fair agreement with previous measurements of the bulk direct and indirect optical band gaps obtained from optical transmission spectroscopy [24].

\section{EXCITON-EXCITON ANNIHILATION IN MONOLAYER MoTe M $_{2}$}

Having introduced monolayer $\mathrm{MoTe}_{2}$ as a direct optical band gap semiconductor with bright near-infrared emission, we now focus on the influence of the exciton formation rate $W_{\text {abs }}$ on its PL quantum yield and PL spectral lineshape under cw laser excitation. $W_{\text {abs }}$ is simply deduced from the effective absorptance of monolayer $\mathrm{MoTe}_{2}$ in the air $/ \mathrm{MoTe}_{2} / \mathrm{SiO}_{2} / \mathrm{Si}$ layered structure, by taking into account the size of our tightly focused laser spot, the absorptance of bare $\mathrm{MoTe}_{2}$ [27] and optical interference effects (see Supplemental Material [53]). For a laser photon energy of $2.33 \mathrm{eV}$, we calculated an absorptance of $\approx 16.5 \%$ for monolayer $\mathrm{MoTe}_{2}$ in our sample geometry. Assuming that one absorbed photon gives rise to one exciton, the exciton formation rates investigated here range from $W_{\text {abs }} \approx 1.0 \times 10^{19} \mathrm{~cm}^{-2} \mathrm{~s}^{-1}$ up to $3.6 \times 10^{22} \mathrm{~cm}^{-2} \mathrm{~s}^{-1}$.

Figure $5(a, b)$ shows PL spectra recorded on the same monolayer for increasing values of $W_{\text {abs }}$. The spectra have been normalized by the incoming laser intensity (i.e., by $W_{\text {abs }}$ ) and by the integration time. We clearly observe a non-linear decrease of the normalized PL intensity that suggests, as shown in Fig. 5(c), that the raw integrated PL intensity levels off with increasing $W_{\text {abs }}$. We have checked that this non-linear behavior was not due to irreversible photo-induced damage of the sample 31] and we have observed a very similar sub-linear rise of the PL intensity on another $\mathrm{MoTe}_{2}$ monolayer (see Supplemental Material [53]). As illustrated in Fig. 5(b), we notice that the linewidth of the PL spectra is independent of $W_{\text {abs }}$ and that the PL spectra downshift very slightly (by only $3 \mathrm{meV}$ ) when $W_{\text {abs }}$ reaches $3.6 \times 10^{22} \mathrm{~cm}^{-2} \mathrm{~s}^{-1}$ (i.e., a laser intensity of $81 \mathrm{~kW} / \mathrm{cm}^{2}$ ). We may thus conclude that biexciton emission [56] and photothermally-induced modifications of the PL spectra can be neglected for the
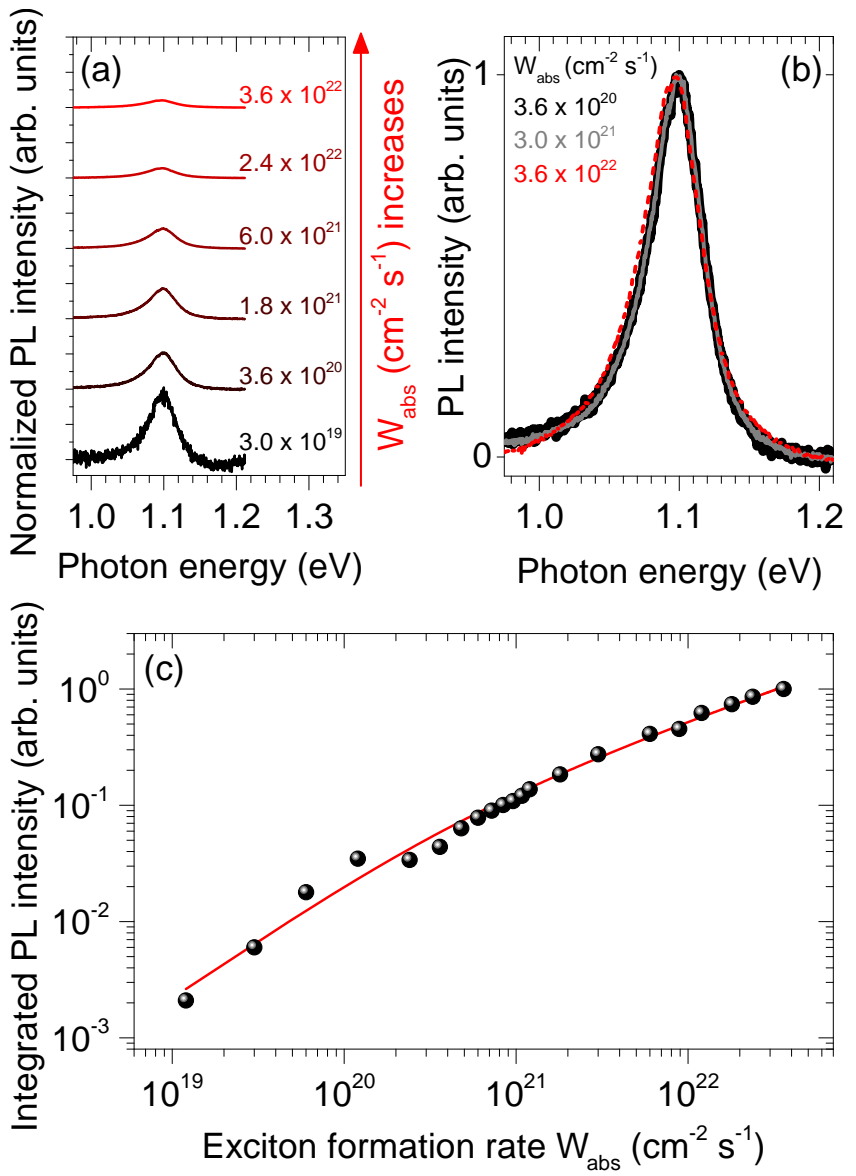

FIG. 5. (a) Photoluminescence spectra of a monolayer $\mathrm{MoTe}_{2}$ sample at different exciton formation rates $W_{\text {abs }}$. The spectra are normalized using the product of $W_{\text {abs }}$ by the integration time and vertically offset for clarity. (b) Photoluminescence spectra of monolayer $\mathrm{MoTe}_{2}$ for three different exciton formation rates. The spectra have been normalized to unity. (c) Integrated photoluminescence intensity obtained from the raw spectra (symbols) as a function of $W_{\text {abs }}$ in monolayer $\mathrm{MoTe}_{2}$. The solid line corresponds to a fit based on Eq. (2). The error bars are smaller than the symbol size.

range of exciton densities explored here.

Sub-linear rises of the integrated PL intensity, as observed in Fig. 5(c), have recently been reported in other $\mathrm{MX}_{2}$ monolayers (such as $\mathrm{WSe}_{2}$ [22, 40, 42, $\mathrm{WS}_{2}$ [22, 41, 42, or $\mathrm{MoS}_{2}$ [42]) and assigned to exciton-exciton annihilation (EEA). EEA has been further evidenced in these materials (and additionally in $\mathrm{MoSe}_{2}$ [39]) by means of transient absorption spectroscopy [38, 39, 42, or timeresolved PL measurements [40, 41], through the observation of accelerated exciton decays at high exciton densities. In order to further demonstrate our observation of EEA in monolayer $\mathrm{MoTe}_{2}$, we make use of a simple rate equation model [42]. The integrated PL intensity is proportional to the steady state exciton density $\left\langle n_{\mathrm{x}}\right\rangle$. Assuming, that the time dependence of the exciton density $n_{\mathrm{x}}$ is essentially governed by the interplay between 
exciton formation (at a rate per unit area $W_{\text {abs }}$ ), linear recombination (at a rate $\Gamma_{\mathrm{x}}$ ) and exciton-exciton annihilation (EEA) (at a rate $\gamma_{\text {eea }}$ ), one obtains

$$
\frac{\mathrm{d} n_{\mathrm{x}}}{\mathrm{d} t}=W_{\mathrm{abs}}-\Gamma_{\mathrm{x}} n_{\mathrm{x}}-\gamma_{\mathrm{eea}} n_{\mathrm{x}}^{2} .
$$

The EEA term in this equation scales quadratically with $n_{\mathrm{x}}$ since the annihilation process involves Coulomb interaction between two excitons. The steady state exciton density is

$$
\left\langle n_{\mathrm{x}}\right\rangle=\frac{\Gamma_{\mathrm{x}}}{2 \gamma_{\text {eea }}}\left(\sqrt{1+\frac{4 \gamma_{\text {eea }}}{\Gamma_{\mathrm{x}}^{2}} W_{\mathrm{abs}}}-1\right) .
$$

The experimental data in Fig. 5(c) is very well fit by Eq. (2). From the fit, we extract $\gamma_{\text {eea }} / \Gamma_{\mathrm{x}}^{2} \approx 1.4 \times$ $10^{-21} \mathrm{~cm}^{2}$ s. Assuming a reasonable value of $\gamma_{\text {eea }} \sim$ $0.1 \mathrm{~cm}^{2} \mathrm{~s}^{-1}$, similar to previous estimates in substratesupported $\mathrm{MX}_{2}$ monolayers 38,42 , one obtains a linear exciton recombination rate of $\Gamma_{\mathrm{x}} \sim 8.5 \times 10^{9} \mathrm{~s}^{-1}$, that is a room temperature exciton lifetime of $\sim 120$ ps. Although additional near-infrared time-resolved measurements or transient absorption studies on monolayer $\mathrm{MoTe}_{2}$ are needed to separately determine the exact values of $\gamma_{\text {eea }}$ and $\Gamma_{\mathrm{x}}$, our simple analysis provides values that are in-line with recent room-temperature measurements on other $\mathrm{MX}_{2}$ [42, 57. Finally, let us also note that monolayer $\mathrm{MoTe}_{2}$ and related systems exhibit EEA rates that give rise to average exciton decay times similar to those reported in carbon nanotubes [58, 59] in the non-linear regime. In addition, EEA in $\mathrm{MX}_{2}$ is much more efficient than related processes (i.e., Auger recombination) in conventional quantum wells [38, 60, 61. Highly efficient EEA between tighly bound excitons [15, 22, 62, 63] in monolayer $\mathrm{MX}_{2}$ reflects the strongly enhanced Coulomb interactions and reduced dielectric screening in these atomically thin two-dimensional materials.

\section{CONCLUSION AND OUTLOOK}

We have performed a detailed analysis of the room temperature photoluminescence of $N$-layer $\mathrm{MoTe}_{2}$.
Monolayer $\mathrm{MoTe}_{2}$ displays a direct optical band gap, with sharp emission at $1.10 \mathrm{eV}$. The crossover from a dominant direct excitonic emission (as observed in monolayers) to a dominant phonon-assisted indirect emission (in the bulk limit) occurs more smoothly than in other $2 \mathrm{Hc}$ transition metal dichalcogenides, such as $\mathrm{MoS}_{2}$, $\mathrm{MoSe}_{2}, \mathrm{WS}_{2}$ and $\mathrm{WSe}_{2}$. As a result, the difference between the bulk indirect optical band gap and the monolayer direct optical band gap is found to be only about $160 \mathrm{meV}$. Our observation of close-lying direct and indirect emission lines invites further calculations of excitonphonon coupling in $\mathrm{MoTe}_{2}$ and related systems, in order to correlate the values of the one-particle indirect band gap to the energy of the emission lines arising from indirect exciton recombination. Interestingly, in bilayer $\mathrm{MoTe}_{2}$, the competition between direct and indirect emission may be efficiently manipulated by external electric fields 64 66, in particular using dual-gated field effect transistors. In addition, we have unveiled a sub-linear scaling of the photoluminescence intensity of monolayer $\mathrm{MoTe}_{2}$ with increasing exciton formation rate, which can be rationalized using a simple model based on exciton-exciton annihilation. This model also allowed us to obtain an order of magnitude estimate for the exciton lifetime in the linear regime that needs to be quantitatively confirmed by time-resolved photoluminescence measurements in the near-infrared range.

\section{ACKNOWLEDGMENTS}

We are grateful to L. Wirtz and A. Molina-Sánchez for stimulating discussions. We thank P. Bernhard at Roper Scientific for the loan of an InGaAs detector. We acknowledge financial support from the Agence Nationale de la Recherche (under grants QuanDoGra 12 JS10-00101 and H2DH ANR-15-CE24-0016), from the LabEx NIE (Under grant WHO), from the CNRS and from Université de Strasbourg.
[1] J.A. Wilson and A.D. Yoffe, "The transition metal dichalcogenides discussion and interpretation of the observed optical, electrical and structural properties," Adv. Phys. 18, 193-335 (1969).

[2] Kin Fai Mak, Changgu Lee, James Hone, Jie Shan, and Tony F. Heinz, "Atomically Thin $\mathrm{MoS}_{2}$ : A New DirectGap Semiconductor," Phys. Rev. Lett. 105, 136805 (2010)

[3] Andrea Splendiani, Liang Sun, Yuanbo Zhang, Tianshu Li, Jonghwan Kim, Chi-Yung Chim, Giulia Galli, and
Feng Wang, "Emerging Photoluminescence in Monolayer $\mathrm{MoS}_{2}, "$ Nano Lett. 10, 1271-1275 (2010)

[4] Changgu Lee, Hugen Yan, Louis E. Brus, Tony F. Heinz, James Hone, and Sunmin Ryu, "Anomalous Lattice Vibrations of Single- and Few-Layer $\mathrm{MoS}_{2}$," ACS Nano 4, 2695-2700 (2010)

[5] A. Molina-Sánchez and L. Wirtz, "Phonons in singlelayer and few-layer $\mathrm{MoS}_{2}$ and $\mathrm{WS}_{2}, "$ Phys. Rev. B 84, $155413(2011)$ 
[6] Guillaume Froehlicher, Etienne Lorchat, François Fernique, Chaitanya Joshi, Alejandro Molina-Sánchez, Ludger Wirtz, and Stéphane Berciaud, "Unified description of the optical phonon modes in $N$-layer $\mathrm{MoTe}_{2}$," Nano Lett. 15, 6481-6489 (2015).

[7] Throughout the text, "direct optical band gap" and "indirect optical band gap" will thus denote the energy of the photons emitted from the direct and indirect bandedge excitons, respectively.

[8] Sefaattin Tongay, Jian Zhou, Can Ataca, Kelvin Lo, Tyler S. Matthews, Jingbo Li, Jeffrey C. Grossman, and Junqiao Wu, "Thermally Driven Crossover from Indirect toward Direct Bandgap in 2D Semiconductors: $\mathrm{MoSe}_{2}$ versus $\mathrm{MoS}_{2}$," Nano Lett. 12, 5576-5580 (2012).

[9] Philipp Tonndorf, Robert Schmidt, Philipp Böttger, Xiao Zhang, Janna Börner, Andreas Liebig, Manfred Albrecht, Christian Kloc, Ovidiu Gordan, Dietrich R. T. Zahn, Steffen Michaelis de Vasconcellos, and Rudolf Bratschitsch, "Photoluminescence emission and Raman response of monolayer $\mathrm{MoS}_{2}, \mathrm{MoSe}_{2}$, and $\mathrm{WSe}_{2}$," Optics Express 21, 4908 (2013).

[10] Weijie Zhao, Zohreh Ghorannevis, Leiqiang Chu, Minglin Toh, Christian Kloc, Ping-Heng Tan, and Goki Eda, "Evolution of electronic structure in atomically thin sheets of $\mathrm{WS}_{2}$ and WSe $2, "$ ACS Nano 7, 791- (2012)

[11] E. Cappelluti, R. Roldán, J. A. Silva-Guillén, P. Ordejón, and F. Guinea, "Tight-binding model and directgap/indirect-gap transition in single-layer and multilayer $\mathrm{MoS}_{2}$," Phys. Rev. B 88, 075409 (2013)

[12] Xiaodong Xu, Wang Yao, Di Xiao, and Tony F. Heinz, "Spin and pseudospins in layered transition metal dichalcogenides," Nat. Phys. 10, 343-350 (2014).

[13] Qing Hua Wang, Kourosh Kalantar-Zadeh, Andras Kis, Jonathan N. Coleman, and Michael S. Strano, "Electronics and optoelectronics of two-dimensional transition metal dichalcogenides," Nat. Nanotechnol. 7, 699-712 (2012)

[14] K. F. Mak, K. L. McGill, J. Park, and P. L. McEuen, "The valley Hall effect in $\mathrm{MoS}_{2}$ transistors," Science 344, 1489-1492 (2014)

[15] Alejandro Molina-Sánchez, Davide Sangalli, Kerstin Hummer, Andrea Marini, and Ludger Wirtz, "Effect of spin-orbit interaction on the optical spectra of singlelayer, double-layer, and bulk $\mathrm{MoS}_{2}$," Phys. Rev. B 88, 045412 (2013)

[16] Diana Y. Qiu, Felipe H. da Jornada, and Steven G. Louie, "Optical spectrum of $\mathrm{MoS}_{2}$ : Many-body effects and diversity of exciton states," Phys. Rev. Lett. 111, 216805 (2013)

[17] Ashwin Ramasubramaniam, "Large excitonic effects in monolayers of molybdenum and tungsten dichalcogenides," Phys. Rev. B 86, 115409 (2012)

[18] Alexey Chernikov, Timothy C. Berkelbach, Heather M. Hill, Albert Rigosi, Yilei Li, Ozgur Burak Aslan, David R. Reichman, Mark S. Hybertsen, and Tony F. Heinz, "Exciton Binding Energy and Nonhydrogenic Rydberg Series in Monolayer $\mathrm{WS}_{2}$," Phys. Rev. Lett. 113, 076802 (2014)

[19] Keliang He, Nardeep Kumar, Liang Zhao, Zefang Wang, Kin Fai Mak, Hui Zhao, and Jie Shan, "Tightly Bound Excitons in Monolayer WSe 2 ," Phys. Rev. Lett. 113, 026803 (2014)

[20] Ziliang Ye, Ting Cao, Kevin OBrien, Hanyu Zhu, Xiaobo Yin, Yuan Wang, Steven G. Louie, and Xiang Zhang,
"Probing excitonic dark states in single-layer tungsten disulphide," Nature 513, 214-218 (2014).

[21] G. Wang, X. Marie, I. Gerber, T. Amand, D. Lagarde, L. Bouet, M. Vidal, A. Balocchi, and B. Urbaszek, "Giant enhancement of the optical second-harmonic emission of $\mathrm{WSe}_{2}$ monolayers by laser excitation at exciton resonances," Phys. Rev. Lett. 114, 097403 (2015).

[22] Bairen Zhu, Xi Chen, and Xiaodong Cui, "Exciton Binding Energy of Monolayer $\mathrm{WS}_{2}$," Scientific Reports 5, 9218 (2015)

[23] Yilei Li, Alexey Chernikov, Xian Zhang, Albert Rigosi, Heather M. Hill, Arend M. van der Zande, Daniel A. Chenet, En-Min Shih, James Hone, and Tony F. Heinz, "Measurement of the optical dielectric function of monolayer transition-metal dichalcogenides: $\mathrm{MoS}_{2}, \mathrm{MoSe}_{2}$, $\mathrm{WS}_{2}$, and WSe 2 ," Phys. Rev. B 90, 205422 (2014).

[24] Ignacio Gutirrez Lezama, Alberto Ubaldini, Maria Longobardi, Enrico Giannini, Christoph Renner, Alexey B. Kuzmenko, and Alberto F. Morpurgo, "Surface transport and band gap structure of exfoliated $2 \mathrm{H}-\mathrm{MoTe}_{2}$ crystals," 2D Mater. 1, 021002 (2014)

[25] Yen-Fu Lin, Yong Xu, Sheng-Tsung Wang, Song-Lin Li, Mahito Yamamoto, Alex Aparecido-Ferreira, Wenwu Li, Huabin Sun, Shu Nakaharai, Wen-Bin Jian, Keiji Ueno, and Kazuhito Tsukagoshi, "Ambipolar $\mathrm{MoTe}_{2}$ Transistors and Their Applications in Logic Circuits," Adv. Mater. 26, 3263-3269 (2014)

[26] A. K. Geim and I. V. Grigorieva, "Van der Waals heterostructures," Nature 499, 419-425 (2013).

[27] Claudia Ruppert, Ozgur Burak Aslan, and Tony F. Heinz, "Optical Properties and Band Gap of Single- and Few-Layer MoTe ${ }_{2}$ Crystals," Nano Lett. 14, 6231-6236 (2014).

[28] Ignacio Gutirrez Lezama, Ashish Arora, Alberto Ubaldini, Cline Barreteau, Enrico Giannini, Marek Potemski, and Alberto F. Morpurgo, "Indirect-to-Direct Band Gap Crossover in Few-Layer $\mathrm{MoTe}_{2}$," Nano Lett. 15, 23362342 (2015)

[29] Jiong Yang, Tieyu Lu, Ye Win Myint, Jiajie Pei, Daniel Macdonald, Jin-Cheng Zheng, and Yuerui Lu, "Robust excitons and trions in monolayer $\mathrm{MoTe}_{2}, "$ ACS Nano 9, 6603-6609 (2015).

[30] Mahito Yamamoto, Sheng Tsung Wang, Meiyan Ni, YenFu Lin, Song-Lin Li, Shinya Aikawa, Wen-Bin Jian, Keiji Ueno, Katsunori Wakabayashi, and Kazuhito Tsukagoshi, "Strong Enhancement of Raman Scattering from a Bulk-Inactive Vibrational Mode in Few-Layer MoTe 2 ," ACS Nano 8, 3895-3903 (2014).

[31] Bin Chen, Hasan Sahin, Aslihan Suslu, Laura Ding, Mariana I. Bertoni, F. M. Peeters, and Sefaattin Tongay, "Environmental changes in $\mathrm{MoTe}_{2}$ excitonic dynamics by defects-activated molecular interaction," ACS Nano 9, 5326-5332 (2015)

[32] Sandhaya Koirala, Shinichiro Mouri, Yuhei Miyauchi, and Kazunari Matsuda, "Homogeneous linewidth broadening and exciton dephasing mechanism in $\mathrm{MoTe}_{2}$," Phys. Rev. B 93, 075411 (2016)

[33] Manabendra Kuiri, Biswanath Chakraborty, Arup Paul, Subhadip Das, A. K. Sood, and Anindya Das, "Enhancing photoresponsivity using $\mathrm{MoTe}_{2}$-graphene vertical heterostructures," Applied Physics Letters 108, 063506 (2016), http://dx.doi.org/10.1063/1.4941996.

[34] Sefaattin Tongay, Hasan Sahin, Changhyun Ko, Alex Luce, Wen Fan, Kai Liu, Jian Zhou, Ying-Sheng Huang, 
Ching-Hwa Ho, Jinyuan Yan, D. Frank Ogletree, Shaul Aloni, Jie Ji, Shushen Li, Jingbo Li, F. M. Peeters, and Junqiao Wu, "Monolayer behaviour in bulk $\mathrm{ReS}_{2}$ due to electronic and vibrational decoupling," Nat. Commun. 5, 3252 (2014).

[35] Huan Zhao, Jiangbin Wu, Hongxia Zhong, Qiushi Guo, Xiaomu Wang, Fengnian Xia, Li Yang, Pingheng Tan, and Han Wang, "Interlayer interactions in anisotropic atomically thin rhenium diselenide," Nano Res. 8, 36513661 (2015)

[36] Daniel Wolverson, Simon Crampin, Asieh S. Kazemi, Adelina Ilie, and Simon J. Bending, "Raman Spectra of Monolayer, Few-Layer, and Bulk ReSe 2 : An Anisotropic Layered Semiconductor," ACS Nano 8, 11154 (2014).

[37] Etienne Lorchat, Guillaume Froehlicher, and Stéphane Berciaud, "Splitting of interlayer shear modes and photon energy dependent anisotropic raman response in $\mathrm{N}$ layer ReSe $\mathrm{R}_{2}$ and $\mathrm{ReS}_{2}$," ACS Nano 10, 27522760 (2016), 10.1021/acsnano.5b07844.

[38] Dezheng Sun, Yi Rao, Georg A. Reider, Gugang Chen, Yumeng You, Louis Brzin, Avetik R. Harutyunyan, and Tony F. Heinz, "Observation of rapid excitonexciton annihilation in monolayer molybdenum disulfide," Nano Lett. 14, 5625-5629 (2014)

[39] Nardeep Kumar, Qiannan Cui, Frank Ceballos, Dawei He, Yongsheng Wang, and Hui Zhao, "Exciton-exciton annihilation in $\mathrm{MoSe}_{2}$ monolayers," Phys. Rev. B 89, 125427 (2014)

[40] Shinichiro Mouri, Yuhei Miyauchi, Minglin Toh, Weijie Zhao, Goki Eda, and Kazunari Matsuda, "Nonlinear photoluminescence in atomically thin layered $\mathrm{WSe}_{2}$ arising from diffusion-assisted exciton-exciton annihilation," Phys. Rev. B 90, 155449 (2014)

[41] Long Yuan and Libai Huang, "Exciton dynamics and annihilation in $\mathrm{WS}_{2} 2 \mathrm{D}$ semiconductors," Nanoscale $\mathbf{7}$, 7402-7408 (2015)

[42] Yiling Yu, Yifei Yu, Chao Xu, Andy Barrette, Kenan Gundogdu, and Linyou Cao, "Fundamental limits of exciton-exciton annihilation for light emission in transition metal dichalcogenide monolayers," Phys. Rev. B 93, 201111 (2016).

[43] K. H. Michel and B. Verberck, "Theory of rigid-plane phonon modes in layered crystals," Phys. Rev. B 85, 094303 (2012)

[44] Yanyuan Zhao, Xin Luo, Hai Li, Jun Zhang, Paulo T. Araujo, Chee Kwan Gan, Jumiati Wu, Hua Zhang, Su Ying Quek, Mildred S. Dresselhaus, and Qihua Xiong, "Interlayer Breathing and Shear Modes in Few-Trilayer $\mathrm{MoS}_{2}$ and $\mathrm{WSe}_{2}$," Nano Lett. 13, 1007-1015 (2013)

[45] P. H. Tan, W. P. Han, W. J. Zhao, Z. H. Wu, K. Chang, H. Wang, Y. F. Wang, N. Bonini, N. Marzari, and N. Pugno, "The shear mode of multilayer graphene," Nat. Mater. 11, 294-300 (2012).

[46] X. Zhang, W. P. Han, J. B. Wu, S. Milana, Y. Lu, Q. Q. Li, A. C. Ferrari, and P. H. Tan, "Raman spectroscopy of shear and layer breathing modes in multilayer $\mathrm{MoS}_{2}$," Phys. Rev. B 87, 115413 (2013)

[47] Mohamed Boukhicha, Matteo Calandra, Marie-Aude Measson, Ophelie Lancry, and Abhay Shukla, "Anharmonic phonons in few-layer $\mathrm{MoS}_{2}$ : Raman spectroscopy of ultralow energy compression and shear modes," Phys. Rev. B 87, 195316 (2013).

[48] M Grzeszczyk, K Goasa, M Zinkiewicz, K Nogajewski, M R Molas, M Potemski, A Wysmoek, and A Babiski,
"Raman scattering of few-layers $\mathrm{MoTe}_{2}$, 2D Materials 3, 025010 (2016)

[49] Q. J. Song, Q. H. Tan, X. Zhang, J. B. Wu, B. W. Sheng, Y. Wan, X. Q. Wang, L. Dai, and P. H. Tan, "Physical origin of Davydov splitting and resonant Raman spectroscopy of Davydov components in multilayer $\mathrm{MoTe}_{2}$," Phys. Rev. B 93, 115409 (2016)

[50] Duhee Yoon, Hyerim Moon, Young-Woo Son, Jin Sik Choi, Bae Ho Park, Young Hun Cha, Young Dong Kim, and Hyeonsik Cheong, "Interference effect on raman spectrum of graphene on $\mathrm{SiO}_{2} / \mathrm{Si}$," Phys. Rev. B 80, $125422(2009)$

[51] Song-Lin Li, Hisao Miyazaki, Haisheng Song, Hiromi Kuramochi, Shu Nakaharai, and Kazuhito Tsukagoshi, "Quantitative raman spectrum and reliable thickness identification for atomic layers on insulating substrates," ACS Nano 6, 7381-7388 (2012)

[52] Michele Buscema, Gary A Steele, Herre SJ van der Zant, and Andres Castellanos-Gomez, "The effect of the substrate on the raman and photoluminescence emission of single-layer $\mathrm{MoS}_{2}, "$ Nano Res. 7, 561-571 (2014).

[53] See Supplemental Material for details on the calculation of the absorptance, optical interference effects, additional Raman scattering data and additional photoluminescence data.

[54] C Robert, R Picard, D Lagarde, G Wang, JP Echeverry, F Cadiz, P Renucci, A Högele, T Amand, X Marie, et al., "Excitonic properties of semiconducting monolayer and bilayer $\mathrm{MoTe}_{2}$," arXiv preprint arXiv:1606.03337 (2016).

[55] Following Refs. 11, 66] the difference between the values of the integrated $\mathrm{PL}$ intensities and of $\mathrm{PL}_{\max }^{-}$recorded in bulk and few-layer flakes $(N=6,7)$ (see Fig. 3 and Fig. (4) may arise from the fact that the bulk conduction band minimum occurs at a point in momentum space that lies halfway between the $\boldsymbol{K}$ and the $\boldsymbol{\Gamma}$ points, while the conduction band minimum is reached at the $\boldsymbol{\Gamma}$ point in the few-layer limit.

[56] Yumeng You, Xiao-Xiao Zhang, Timothy C. Berkelbach, Mark S. Hybertsen, David R. Reichman, and Tony F. Heinz, "Observation of biexcitons in monolayer $\mathrm{WSe}_{2}$," Nat. Phys. 11, 477-481 (2015).

[57] C. Robert, D. Lagarde, F. Cadiz, G. Wang, B. Lassagne, T. Amand, A. Balocchi, P. Renucci, S. Tongay, B. Urbaszek, and X. Marie, "Exciton radiative lifetime in transition metal dichalcogenide monolayers," Phys. Rev. B 93, 205423 (2016)

[58] Ying-Zhong Ma, Leonas Valkunas, Susan L. Dexheimer, Sergei M. Bachilo, and Graham R. Fleming, "Femtosecond spectroscopy of optical excitations in single-walled carbon nanotubes: Evidence for exciton-exciton annihilation," Phys. Rev. Lett. 94, 157402 (2005)

[59] Feng Wang, Gordana Dukovic, Ernst Knoesel, Louis E. Brus, and Tony F. Heinz, "Observation of rapid auger recombination in optically excited semiconducting carbon nanotubes," Phys. Rev. B 70, 241403 (2004)

[60] A Haug, "Auger recombination in quantum well semiconductors: Calculation with realistic energy bands," Semiconductor Science and Technology 7, 1337 (1992).

[61] R.A. Taylor, R.A. Adams, J.F. Ryan, and R.M. Park, "Exciton recombination dynamics in ZnCdSe/ZnSe quantum wells," Journal of Crystal Growth 159, 822 825 (1996)

[62] A. R. Klots, A. K. M. Newaz, Bin Wang, D. Prasai, H. Krzyzanowska, Junhao Lin, D. Caudel, N. J. Ghimire, 
J. Yan, B. L. Ivanov, K. A. Velizhanin, A. Burger, D. G. Mandrus, N. H. Tolk, S. T. Pantelides, and K. I. Bolotin, "Probing excitonic states in suspended two-dimensional semiconductors by photocurrent spectroscopy," Scientific Reports 4, 6608 (2014)

[63] Miguel M. Ugeda, Aaron J. Bradley, Su-Fei Shi, Felipe H. da Jornada, Yi Zhang, Diana Y. Qiu, Wei Ruan, Sung-Kwan Mo, Zahid Hussain, Zhi-Xun Shen, Feng Wang, Steven G. Louie, and Michael F. Crommie, "Giant bandgap renormalization and excitonic effects in a monolayer transition metal dichalcogenide semiconductor," Nat. Mater. 5, 1091 (2014).

[64] Ashwin Ramasubramaniam, Doron Naveh, and Elias Towe, "Tunable band gaps in bilayer transition-metal dichalcogenides," Phys. Rev. B 84, 205325 (2011).

[65] Nourdine Zibouche, Pier Philipsen, Agnieszka Kuc, and Thomas Heine, "Transition-metal dichalcogenide bilayers: Switching materials for spintronic and valleytronic applications," Phys. Rev. B 90, 125440 (2014).

[66] Thomas Brumme, Matteo Calandra, and Francesco Mauri, "First-principles theory of field-effect doping in transition-metal dichalcogenides: Structural properties, electronic structure, hall coefficient, and electrical con- ductivity," Phys. Rev. B 91, 155436 (2015).

[67] Enze Zhang, Yibo Jin, Xiang Yuan, Weiyi Wang, Cheng Zhang, Lei Tang, Shanshan Liu, Peng Zhou, Weida $\mathrm{Hu}$, and Faxian $\mathrm{Xiu}$, $\mathrm{ReS}_{2}$-based field-effect transistors and photodetectors," Adv. Func. Mater. 25, 4076-4082 (2015)

[68] Eugene Hecht, Optics ( ${ }^{\text {th }}$ Edition) (Addison-Wesley, 2001).

[69] A.R. Beal and H.P. Hughes, "Kramers-Kronig analysis of the reflectivity spectra of $2 \mathrm{H}-\mathrm{MoS}_{2}, 2 \mathrm{H}-\mathrm{MoSe}_{2}$ and $2 \mathrm{H}-$ $\mathrm{MoTe}_{2}, "$ J. Phys. C: Solid State Phys. 12, 881 (1979).

[70] I. H. Malitson, "Interspecimen comparison of the refractive index of fused silica," J. Opt. Soc. Am. 55, 1205-1209 (1965)

[71] Edward D. Palik, Handbook of Optical Constants of Solids (Elsevier, 1998) pp. $561-565$.

[72] Th. Böker, R. Severin, A. Müller, C. Janowitz, R. Manzke, D. Voß, P. Krüger, A. Mazur, and J. Pollmann, "Band structure of $\mathrm{MoS}_{2}, \mathrm{MoSe}_{2}$, and $\alpha-\mathrm{MoTe}_{2}$ : Angle-resolved photoelectron spectroscopy and $a b$ initio calculations," Phys. Rev. B 64, 235305 (2001). 


\section{Supplemental Material}

SI 1. HIGH-FREQUENCY RAMAN SPECTRA

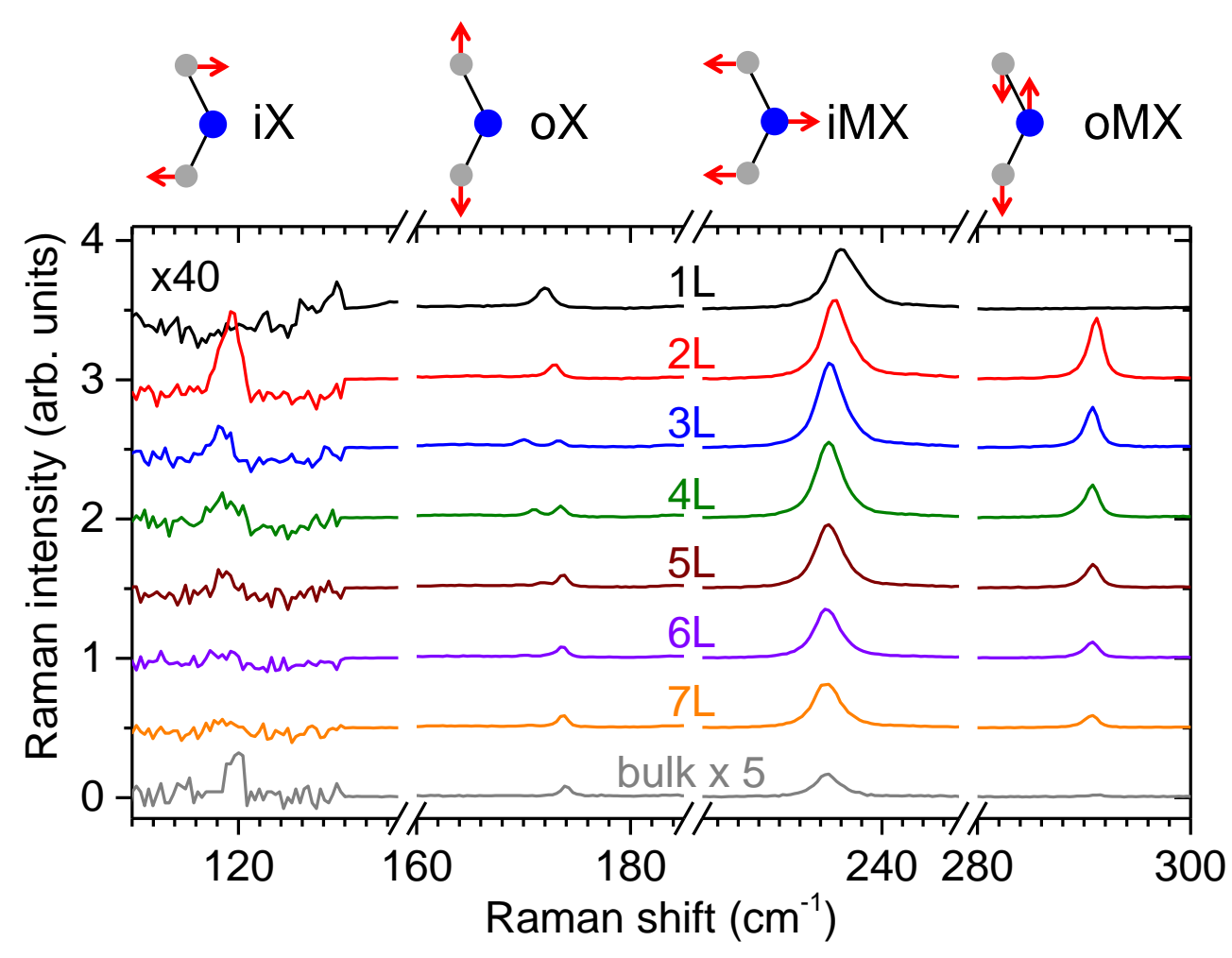

FIG. S1. High-frequency Raman spectra of $N=1$ to $N=7$ layers $\mathrm{MoTe}_{2}$ and of bulk $\mathrm{MoTe}_{2}$. The spectra are vertically offset for clarity. The four one-phonon features are labeled and the atomic displacements for the monolayer are indicated with Mo atoms in blue and Te atoms in grey.

Figure S1 shows the high-frequency range $\left(100-300 \mathrm{~cm}^{-1}\right)$ of the Raman spectra of Fig. 1(c) in the manuscript. We observe the four expected one-phonon features assigned to intralayer displacements in $2 H c$ transition metal dichalcogenides [6] 67]: (i) the in-plane, out-of-phase vibration of the Te planes, with $\mathrm{E}_{1 g}$ symmetry in bulk (iX mode at $120 \mathrm{~cm}^{-1}$ ), (ii) the out-of-plane, out-of-phase vibration of the Te planes, with $\mathrm{A}_{1 g}$ symmetry in bulk (oX mode at $170 \mathrm{~cm}^{-1}$ ), (iii) the in-plane vibration of the Mo and Te planes against each other, with $\mathrm{E}_{2 g}$ symmetry in bulk (iMX mode at $235 \mathrm{~cm}^{-1}$ ), and (iv) the out-of-plane vibration of the Mo and Te planes against each other, with $\mathrm{B}_{2 g}$ symmetry in bulk (oMX mode at $290 \mathrm{~cm}^{-1}$ ). Note that we discern the Davydov splitting of the oX mode as recently reported in Refs. 6, 48, 49, confirming the number of layers $N$ deduced from the low-frequency part of the spectra. 
SI 2. LASER SPOT AREA

(a)

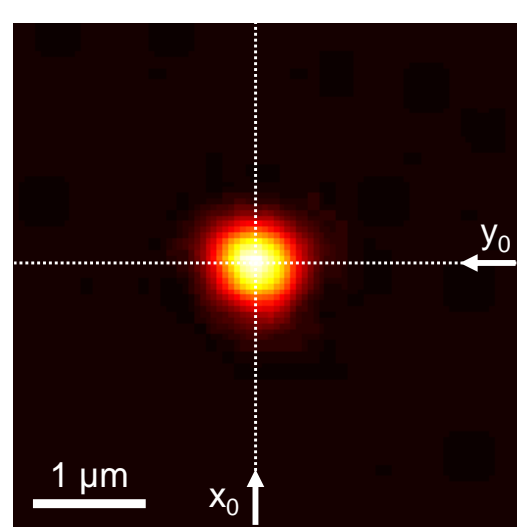

(c)

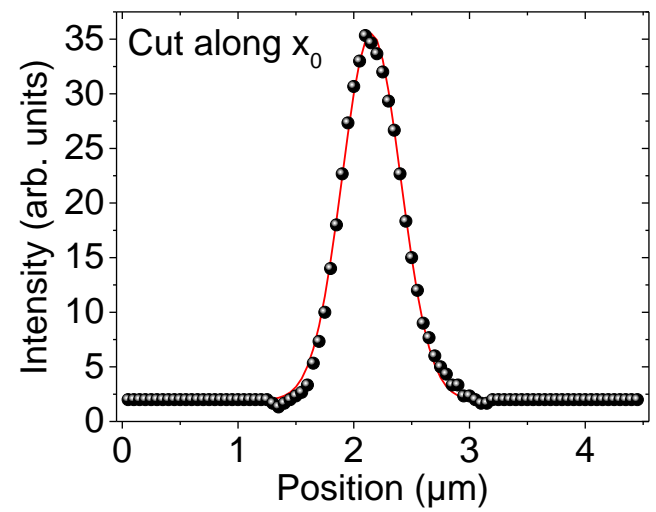

(b)

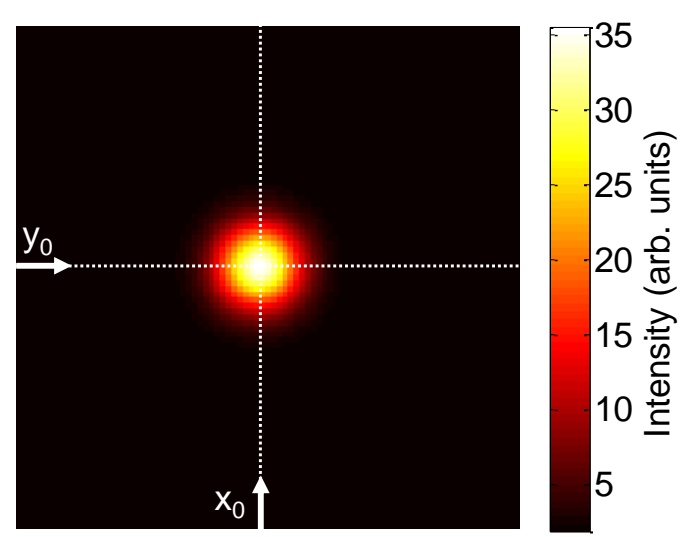

(d)

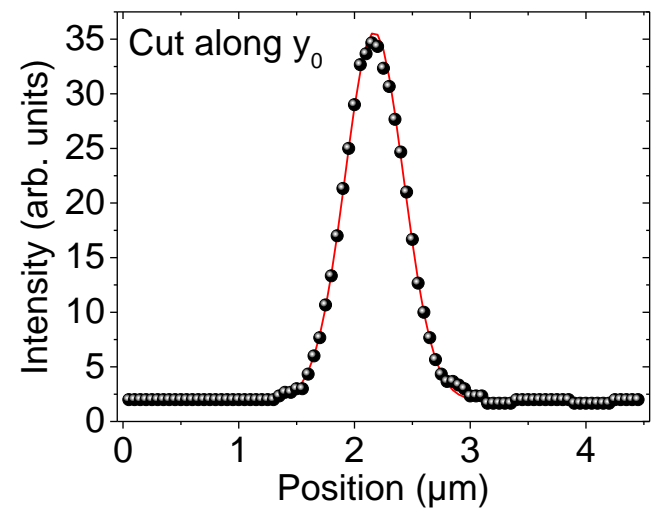

FIG. S2. (a) Optical image of the focused laser spot on the surface of a substrate. (b) Two-dimensional fit of the optical image. Cuts along (c) $x_{0}$ and (d) $y_{0}$. The solid lines are the fit to the experimental data (symbols).

In order to measure the area of our laser spot, we recorded an optical image of the tightly focused laser spot on the surface of a substrate (see Fig. S2(a)). We have then fitted this image with a two-dimensional Gaussian function

$$
f(x, y)=A \exp \left(-\frac{\left(x-x_{o}\right)^{2}+\left(y-y_{o}\right)^{2}}{2 \sigma^{2}}\right),
$$

where $A$ is the amplitude of the Gaussian, $\left(x_{0}, y_{0}\right)$ are the coordinates of the center and $\sigma$ is the standard deviation (we assumed that the standard deviation is the same for the two dimensions). On Fig. S2(b)-(d), we observe that the data are well fitted by this function. Knowing that the surface area is given by $2 \pi \sigma^{2}$, we deduced a laser spot area of $4 \times 10^{-9} \mathrm{~cm}^{2}$ that has been used to estimate the exciton formation rate per unit area $W_{\text {abs }}$ in the main manuscript. 


\section{SI 3. INTERFERENCE EFFECTS}

It is well-known that interference effects strongly affect optical absorption, as well as the Raman [50, 51, and PL [52] signal of layered materials. Indeed, multiple reflections at different interfaces (air/ $/ \mathrm{MoTe}_{2}, \mathrm{MoTe}_{2} / \mathrm{SiO}_{2}$ and $\mathrm{SiO}_{2} / \mathrm{Si}$ ) can enhance the absorption of the incoming light beam (see Fig. S3 and Fig. S4(a)) as well as the PL (or Raman) intensity (see Fig. S4 (b)) by a factor $\mathrm{F}_{\mathrm{ab}}$ and $\mathrm{F}_{\mathrm{pl}}$, respectively.

\section{SI 3a. Absorptance of the monolayer $\mathrm{MoTe}_{2}$}

We first consider the absorptance of $N$-layer $\mathrm{MoTe}_{2}$. Due to interference effects in the air $/ \mathrm{MoTe}_{2} / \mathrm{SiO}_{2} / \mathrm{Si}$ structure, the absorptance $A$ (absorbed fraction of incident light) of $N$-layer $\mathrm{MoTe}_{2}$ is different as compared to the freestanding case. As it is drawn in Fig. S3, a fraction $R$ (reflectance) of the incident excitation light is reflected, a fraction $T$ (transmittance) is transmitted into the Si substrate, which is supposed to be semi-infinite (in practice the substrate only needs to be ticker than a few absorption lengths) and a fraction A is absorbed. Energy conservation imposes 68.

$$
A+R+T=1 .
$$

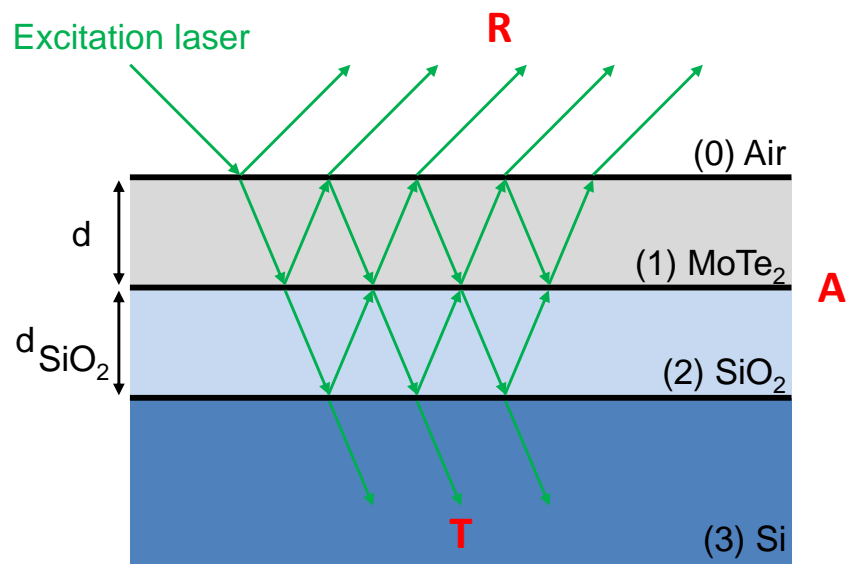

FIG. S3. Schematic diagrams of the optical paths in our geometry for a laser photon energy of $2.33 \mathrm{eV}$. $R$ is the reflectance, $T$ the transmittance and $A$ the absorptance.

Since $\mathrm{SiO}_{2}$ is supposed to be transparent, the absorptance of $N$-layer $\mathrm{MoTe}_{2}$ in this structure is directly given by $A=1-R-T$. $R$ and $T$ can be obtained analytically using an interference calculation [50, 51. Without changing drastically the results, we can assume that the light impinges on the sample at normal incidence. In this condition, the Fresnel coefficients are $t_{i j}=2 n_{i} /\left(n_{i}+n_{j}\right)$ and $r_{i j}=\left(n_{i}-n_{j}\right) /\left(n_{i}+n_{j}\right) . n_{0}=1$ is the refractive index of air and $n_{1}, n_{2}$ and $n_{3}$ are the complex refractive index for $\mathrm{MoTe}_{2}, \mathrm{SiO}_{2}$ and $\mathrm{Si}$, respectively. The phase factors are $\beta_{1}=2 \pi n_{1} d / \lambda$ and $\beta_{2}=2 \pi n_{2} d_{\mathrm{SiO}_{2}} / \lambda$ where $\lambda$ is the wavelength of the light in vacuum and $d\left(d_{\mathrm{SiO}_{2}}\right)$ is the thickness of $\mathrm{MoTe}_{2}\left(\mathrm{SiO}_{2}\right)$. Thus, the normal incidence reflectance $R$ and transmittance $T$ are given by 68 .

$$
\begin{aligned}
& R=\left|\frac{r_{01}\left[1+r_{12} r_{23} e^{2 i \beta_{2}}\right]+\left[r_{12}+r_{23} e^{2 i \beta_{2}}\right] e^{2 i \beta_{1}}}{1+r_{12} r_{23} e^{2 i \beta_{2}}+\left[r_{12}+r_{23} e^{2 i \beta_{2}}\right] r_{01} e^{2 i \beta_{1}}}\right|^{2}, \\
& T=\left|\frac{t_{01} t_{12} t_{23} t_{32} t_{21} t_{10} e^{2 i\left(\beta_{1}+\beta_{2}\right)}}{\left(1+r_{12} r_{23} e^{2 i \beta_{2}}+\left[r_{12}+r_{23} e^{2 i \beta_{2}}\right] r_{01} e^{2 i \beta_{1}}\right)^{2}}\right| .
\end{aligned}
$$

At a photon energy of $2.33 \mathrm{eV}$, the refractive index of $\mathrm{MoTe}_{2}$ is $n_{2.33 \mathrm{eV}}=4.07+1.63 i$ [69, of $\mathrm{SiO}_{2} n_{\mathrm{SiO}_{2}}=1.4607$ [70] and of $\mathrm{Si} n_{\mathrm{Si}}=4.14+0.045 i$ [71]. For a monolayer $\mathrm{MoTe}_{2}$ of thickness $c / 2=0.6984 \mathrm{~nm}$ [72] and a $\mathrm{SiO}_{2}$ layer of $d_{\mathrm{SiO}_{2}}=90 \mathrm{~nm}$, we calculated an absorptance $A \approx 16.5 \%$, which is in line with the absorptance of other TMDs measured on $\mathrm{SiO}_{2} / \mathrm{Si}[9]$. 


\section{SI 3b. Normalization process}

In order to quantitatively compare the PL spectra recorded on $N$-layer $\mathrm{MoTe}_{2}$, one has to take into account (i) the response of the setup (grating and camera) and (ii) the optical interference and absorption effects. The response of the camera is supposed to be flat (it varies by less than 5\%) in the spectral range studied here. The response of the grating is extracted from the data of the manufacturer (Richardson Gratings 53-*-500R).

(a)

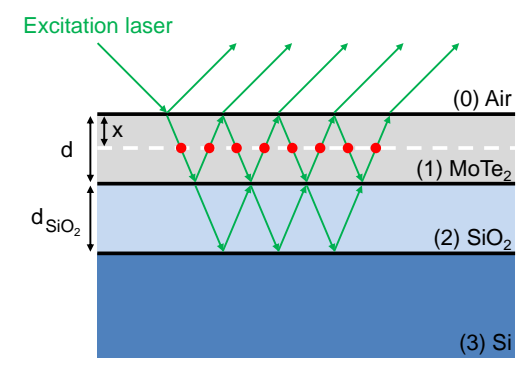

(c)

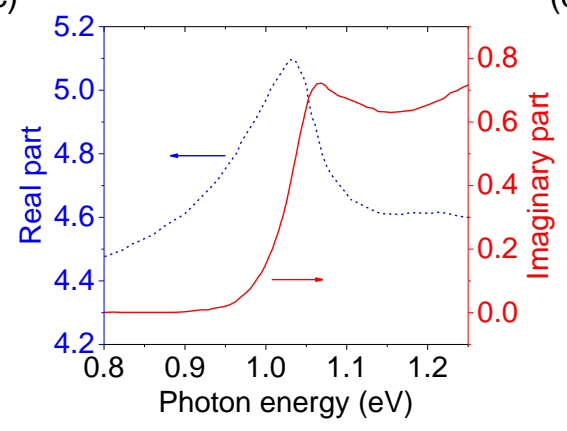

(b)

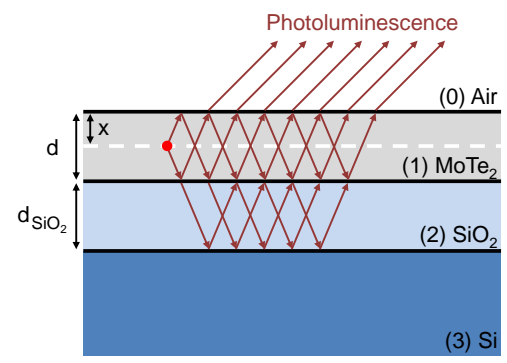

(d)

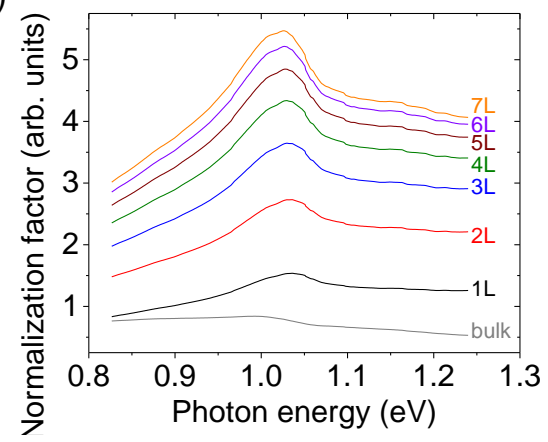

FIG. S4. Schematic diagram of the optical paths in our geometry for (a) the excitation laser (at a photon energy of 2.33 eV) and (b) the emitted light. $d$ is proportional to $N$.(c) Refractive index of $\mathrm{MoTe}_{2}$, extracted from the measurements in Ref. 24, as a function of the emission energy. (d) Normalization factor as function of the emission energy.

Following the results reported in Refs. [50, 51], we calculated the enhancement factor for the PL due to the multiple interference

$$
F=\int_{0}^{d}\left|F_{\mathrm{ab}}(x) F_{\mathrm{pl}}(x)\right|^{2} \mathrm{~d} x
$$

with

$$
\begin{gathered}
F_{\mathrm{ab}}=t_{01} \frac{\left[1+r_{12} r_{23} e^{2 i \beta_{2}}\right] e^{i \beta_{x}}+\left[r_{12}+r_{23} e^{2 i \beta_{2}}\right] e^{i\left(2 \beta_{1}-\beta_{x}\right)}}{1+r_{12} r_{23} e^{2 i \beta_{2}}+\left[r_{12}+r_{23} e^{2 i \beta_{2}}\right] r_{01} e^{2 i \beta_{1}}} \\
F_{\mathrm{pl}}=t_{10} \frac{\left[1+r_{12} r_{23} e^{2 i \beta_{2}}\right] e^{i \beta_{x}}+\left[r_{12}+r_{23} e^{2 i \beta_{2}}\right] e^{i\left(2 \beta_{1}-\beta_{x}\right)}}{1+r_{12} r_{23} e^{2 i \beta_{2}}+\left[r_{12}+r_{23} e^{2 i \beta_{2}}\right] r_{01} e^{2 i \beta_{1}}}
\end{gathered}
$$

where we used exactly the same notations as previously. $\beta_{x}=2 \pi n_{1} x / \lambda$ with $x$ being the depth of the point where the interactions occur (see Fig. S4(a)-(b)). Note that the expressions for $\mathrm{F}_{\mathrm{ab}}$ and $\mathrm{F}_{\mathrm{pl}}$ are similar, but the wavelengths in the phase factors are different.

For the excitation laser, we used the same refractive index as for the absorptance calculations. For the PL, we used the values extracted from the dielectric function measured in Ref. [24] (see Fig. S4(c)) for $\mathrm{MoTe}_{2}$, and the tabulated values for $\mathrm{SiO}_{2}$ and $\mathrm{Si}$ from Refs. [70] and [71, respectively. We also used $d=N \times c / 2$, where $c / 2=0.6984 \mathrm{~nm}[72$ ] is the thickness of one layer.

By multiplying the enhancement factors and the grating response, we obtained the normalized factors plotted in Fig. S4 (d). Note that for the bulk, we supposed that $\mathrm{MoTe}_{2}$ is semi-infinite, i.e., there is only one interface, air $/ \mathrm{MoTe}_{2}$. Finally, we divided the PL spectra by the corresponding normalization factor. 


\section{SI 4. PL SPECTRA AT TWO DIFFERENT PHOTON ENERGIES}

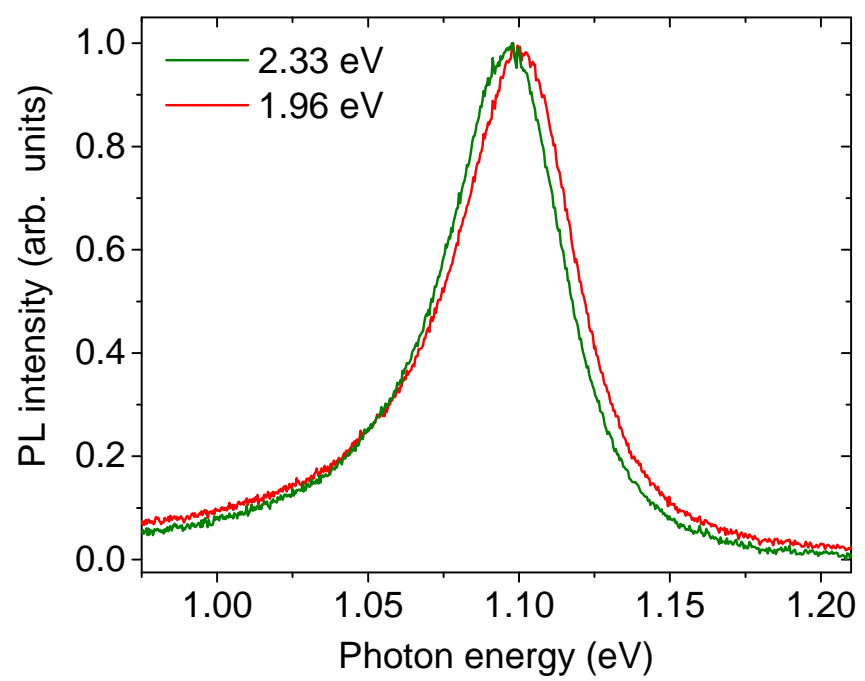

FIG. S5. Photoluminescence spectra of a monolayer $\mathrm{MoTe}_{2}$ recorded under the same condition at laser photon energies of $2.33 \mathrm{eV}$ and $1.96 \mathrm{eV}$. When exciting at $1.96 \mathrm{eV}$, we observe that the emission energy blueshifts by $\approx 6$ meV compared to a reference spectrum recorded using a laser excitation at $2.33 \mathrm{eV}$. However, we do not observe significant modifications of the PL lineshape.

SI 5. PHOTOLUMINESCENCE OF THE SI SUBSTRATE

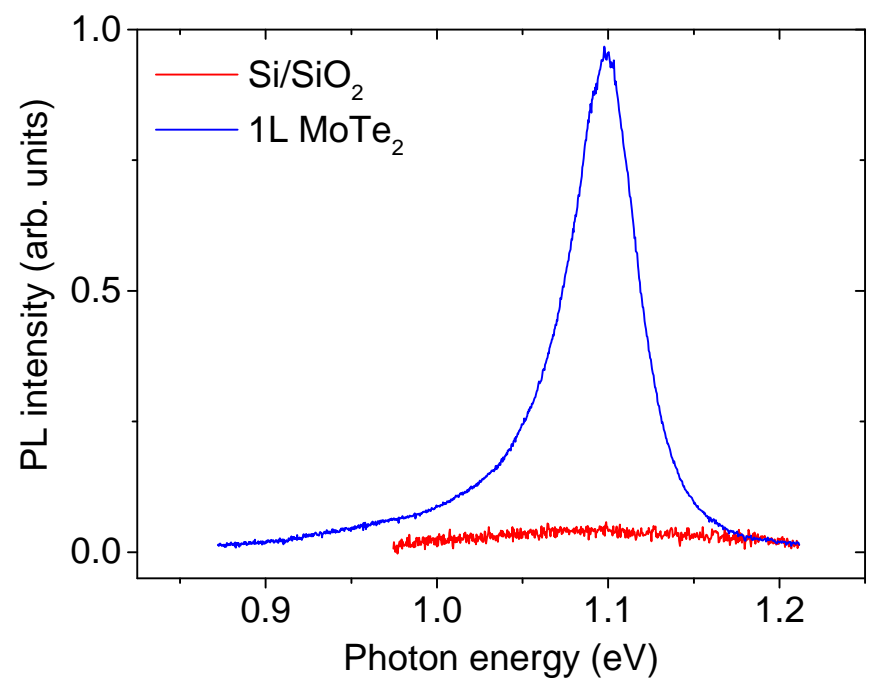

FIG. S6. Photoluminescence spectra of a monolayer $\mathrm{MoTe}_{2}$ and of the bare $\mathrm{Si} / \mathrm{SiO}_{2}$ substrate recorded at $2.33 \mathrm{eV}$ under a laser intensity of $6.5 \mathrm{~kW} \mathrm{~cm}^{-2}$ (corresponding to an exciton formation rate of $W_{\mathrm{abs}} \approx 2.9 \times 10^{21} \mathrm{~cm}^{-2} \mathrm{~s}^{-1}$ in monolayer MoTe 2 ). The emission from the substrate is negligible in our study. 


\section{SI 6. EXCITON-EXCITON ANNIHILATION ON ANOTHER SAMPLE}

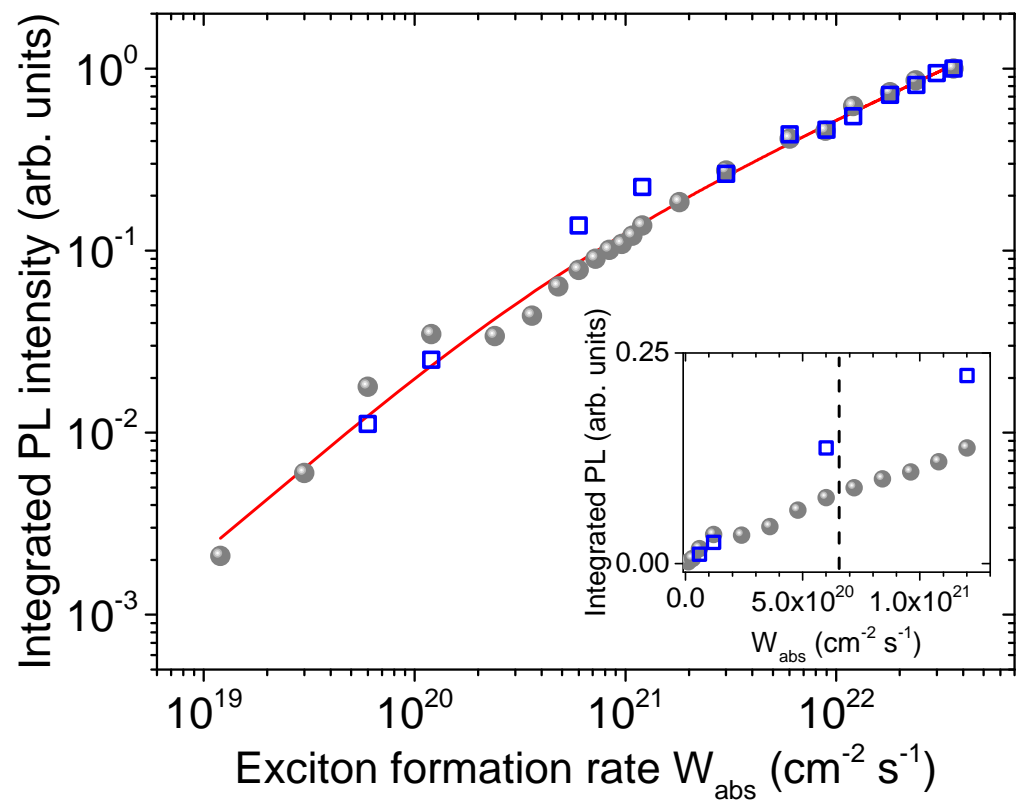

FIG. S7. Integrated photoluminescence intensity as a function of the exciton formation rate $W_{\text {abs }}$ for two different monolayer samples of $\mathrm{MoTe}_{2}$. The gray-filled circles are the same data as Fig. 5(c) in the manuscript (sample 1). The solid red line is the fit to this data using Eq. (2), as in the manuscript. The raw PL intensity from the second sample (sample 2) has been multiplied by a factor of 2.4 (open blue squares) to show a clearer comparison with the data from sample 1 . The normalized PL intensity from sample 2 scales very similarly as the PL intensity from sample 1, suggesting similar exiton-exciton annihilation rates and linear exciton decay rates for both samples. The inset shows the same data at low exciton formation rates $W_{\text {abs }}$, on a linear scale. The vertical dashed line indicates the excitation formation rate at which the measurements on $N$-layer MoTe 2 shown in Fig. 2 and 3 of main manuscript have been performed. The error bars are smaller than the symbol size. 